\title{
Assessing circular RNAs in Alzheimer disease
}

Laura Cervera-Carles ${ }^{1,2}$, Oriol Dols-Icardo ${ }^{1,2}$, Alba Cervantes-Gonzalez ${ }^{1}$, Laura Molina-Porcel ${ }^{3}$, Laia Muñoz-Llahuna ${ }^{1,2}$, and Jordi Clarimon ${ }^{1,2}$

${ }^{1}$ Genetics of Neurodegenerative Disorders Unit, Sant Pau Biomedical Research Institute, Hospital de la Santa Creu i Sant Pau, Universitat Autònoma de Barcelona, Barcelona, Spain.

${ }^{2}$ CIBERNED, Center for Networked Biomedical Research into Neurodegenerative Diseases, Madrid, Spain.

${ }^{3}$ Neurological Tissue Bank of the Biobanc-Hospital Clinic-IDIBAPS, Institut d'Investigacions Biomèdiques August Pi I Sunyer, Barcelona, Spain.

\section{Corresponding author:}

Jordi Clarimon, PhD

Genetics of Neurodegenerative Disorders Unit

Sant Pau Research Institute

Sant Quintí, 77-79

08041 Barcelona, Spain

Phone: 34932909050 ext. 8240

e-mail: jclarimon@santpau.cat

\section{Keywords:}

Alzheimer's disease; circRNA; noncoding RNA; genetics; frontotemporal lobar degeneration. 


\section{Abstract}

Circular RNAs (circRNA) are evolutionary conserved non-coding RNAs resulting from the backsplicing of precursor messengers. Recently, a circular-transcriptome-wide study of circRNA in brain tissue from patients with Alzheimer's disease (AD) has revealed a striking association between the expression of circRNA and AD pathological diagnosis. In the present study, we aimed at replicating the major findings in an independent case series comprising definitive sporadic and familial AD. In order to assess the specificity of circRNA changes, we also included cases with frontotemporal lobar degeneration (FTLD), comprising brain specimens with TDP-43 aggregates (FTLD-TDP43) and samples that presented Tau accumulation (FTLD-Tau). Through a quantitative PCR approach, we evaluated a total of eight circRNAs that surpassed the significant threshold in the former meta-analysis (cirCHOMER1, cirCDOCK1, cirCKCNN2, cirCMAN2A1, cirCFMN1, circRTN4, circMAP7, and circPICALM). Average expression changes between $A D$ patients and controls followed the same directions as previously reported, suggesting an overall upregulation of cirCDOCK1, cirCMAP7, cirCMAN2A1, cirCRTN4 and $\operatorname{cirCPICALM,~and~a~downregulation~of~the~}$ remainder (cirCHOMER1, cirCFMN1 and cirCKCNN2) in AD brain tissue. We also confirmed an exacerbated alteration in circRNA expression in the Mendelian AD group compared to the sporadic forms. Two circRNAs, circHOMER1 and circKCNN2, also showed significant expression alterations in the group of FTLD-Tau and FTLD-TDP43, respectively. Overall, these results reinforce the conception that expression of circRNAs is altered in Alzheimer's disease, and also suggest a wider involvement of this particular class of RNA in other neurodegenerative dementias. 


\section{Introduction}

Circular RNAs (circRNAs) are a class of low-abundance biochemically stable single stranded RNAs, produced by the back-splicing of exons in precursor mRNAs. Although their role in the central nervous system is poorly understood, the enriched expression in brain tissue and their overall upregulation during neurogenesis and synaptic plasticity has fueled the interest of these noncoding family of transcripts to underscore the biological bases of neurodegenerative disorders ${ }^{1-3}$

Recently, using deep RNA profiling in brain tissue from patients with Alzheimer disease (AD) and healthy controls, Dube et al. have shown evidence for changes in circRNA expression occurring early, in pre-symptomatic AD, as well as in Mendelian forms of the disease ${ }^{4}$. Through a circular transcriptome-wide analysis in two independent RNA-seq datasets, the authors disclosed more than 3.500 different circRNA species. Among them, 28 were significantly associated with the three traits that were investigated: neuropathological staging, dementia severity and differential expression between phenotypes. Correction for multiple comparisons disclosed a group of circRNAs that surpassed the p-value threshold for all three traits in their meta-analysis. In the present study we aimed at replicating some of the observed changes in an independent cohort of patients with a neuropathological diagnosis and assess whether these alterations are specific to AD.

\section{Methods}

Total RNA was isolated from $60 \mathrm{mg}$ of frozen frontal cortex samples obtained from the Neurological Tissue Bank (Barcelona). Brain tissue was first homogenized with a mortar and pestle under liquid nitrogen and transferred into Trizol reagent (Life Technologies). The conventional 
phenol-chloroform protocol was followed by a column-based RNA purification method using the RNeasy Mini kit (Qiagen, Hilden, Germany). RNA integrity (RIN) was assessed using the 2100 Bioanalyzer instrument (Agilent). All RNA samples presented a RIN $>6$.

RNA was reverse transcribed with the RevertAid First Strand cDNA synthesis kit (Thermo Scientific, Rockford, IL). For quantitative polymerase chain reaction (qPCR), the obtained cDNAs were analyzed in duplicate in a 7900HT Fast Real-Time PCR system (Applied Biosystems, Foster City, CA), using the Fast SYBR Green Master mix (Thermo Scientific), $200 \mathrm{nM}$ of each primer and $30 \mathrm{ng}$ of cDNA. circRNAs were assayed using the previously reported primers ${ }^{4}$, and GAPDH was used as normalizer. Prior to the analysis a detection threshold for qPCR was established at 38 cycles. Expression levels were determined by the $2^{-\Delta \Delta c t}$ method. Kruskal-Wallis non-parametric test with Dunn's post-hoc analysis was applied for comparisons between diagnostic groups. Adjusted statistical significance was set at $\alpha=0.05$.

Neuropathological examination was performed according to standardized protocols at the Neurological Tissue Bank of the Biobanc-Hospital Clinic-IDIBAPS ${ }^{5}$, and disease evaluation was performed according to international consensus criteria ${ }^{6,7}$.

\section{Results}

In the present study we assessed the expression of 8 circRNA species that exceeded the stringent gene-based, Bonferroni multiple test correction in the final meta-analysis by Dube et al.: cirCHOMER1, cirCDOCK1, cirCKCNN2, cirCMAN2A1, cirCFMN1, cirCRTN4, cirCMAP7, and circPICALM. The frontal cortex of 69 brain samples was included in the analysis (Table 1). These included samples from patients with a definitive diagnosis of $A D(n=19)$, patients with frontotemporal 
lobar degeneration (FTLD) presenting aggregates of the transactive response DNA-binding protein $43 \mathrm{kDa}$ (FTLD-TDP43, $\mathrm{n}=16$ ), and patients with Tau pathology (FTLD-Tau, 6 with corticobasal degeneration and 4 with Pick's disease, Table 1). Since individuals with autosomal dominant AD showed more dramatic changes in circRNA expression compared to sporadic AD in the Dube et al. study, we also added 9 brain specimens from familial AD patients harboring Mendelian mutations (fAD) (see Table 1 for details). Similarly, among the FTLD series 9 individuals harbored the hexanucleotide repeat expansion in the C9ORF72 gene (a known genetic cause of FTLD-TDP) and 5 were carriers of the p.P301L missense mutation in the MAPT gene (leading to FTLD-Tau). Fifteen postmortem brain samples from neurologically healthy controls were also included. All samples were provided by the Neurological Tissue Bank at Hospital Clínic (IDIBAPS, Barcelona), and met the neuropathological diagnostic criteria for their respective diagnoses ${ }^{6,7}$. The study (IIBSP-DFT-201897) was approved by the local Ethics Committee of both the Tissue Bank and Sant Pau Research Institute.

Our evaluation through qPCR using divergent oligonucleotides revealed direction effects that were consistent with those reported by Dube et al. in the context of AD, with cirCHOMER1, circKCNN2, cirCFMN1 and cirCMAN2A1 showing a trend towards a decreased expression, and circDOCK1, circMAP7, cirCMAN2A1, circRTN4, and circPICALM presenting a tendency towards an enriched expression in AD patients compared to controls (Figure 1). However, only in the case of cirCDOCK1 and circHOMER1 these differences reached statistical significance in the sporadic AD (SAD) group. Notably, in accordance with Dube et al. work, the group of fAD, conformed by subjects with Mendelian forms of disease, presented more striking differences in the levels of circRNAs compared to controls than the non-genetic forms of the disease, with $4 / 8$ circRNAs showing significant differences between $F A D$ and controls. 
In order to assess whether these changes were specific to $A D$, we evaluated the expression pattern of these circRNAs in FTLD. The levels of circKCNN2 were significantly reduced in the FTLDTDP43 group compared to healthy controls. Among FTLD-Tau, levels of circHOMER1 were also decreased compared to controls. When genetic causes of FTLD due to mutations in C9ORF72 or MAPT were analyzed independently, we did not find any significant change in the expression of any circRNAs compared to controls (data not shown). Also, no correlation of circRNA levels with the age of death was found in the whole sample, or when the analysis was stratified by the 5 phenotype groups (data not shown).

\section{Discussion}

Overall, our results reinforce and complement the findings by Dube and collaborators, showing that the levels of some circRNAs are altered in brain tissue of AD patients. Interestingly, our results in the autosomal-dominant $A D$ group revealed even larger changes in circRNA compared to the sporadic counterpart, confirming previous results. Our correlation analyses partially precluded a possible age-related effect that could influence these outcomes. However, it is important to note that our fAD series was significantly younger than controls. Perfectly age-matched controls with younger age at death to compare circRNA levels with the fAD group would be an invaluable resource to better understand these substantial differences in this particular form of disease. The inclusion of patients with FTLD in our study revealed that some significant changes in circRNA levels can also be observed in other neurodegenerative disorders. A good example is the significant decrease of the expression levels of circHOMER1 in FTLD-Tau. The presence of cirCHOMER1 in neuronal cell bodies and dendrites, its substantial expression in the hippocampus (a region particularly vulnerable in $A D$ and other dementias), and the alterations of its expression 
levels due to neuronal activity ${ }^{1}$, strengthens its role (and maybe the role of other circRNAs with similar behaviors) across different neurodegenerative disorders.

Although recently discovered, these heterogeneous groups of transcripts are very promising candidates to study in order to better understand the biological bases of neurodegenerative disorders, discover novel peripheral biomarkers and may give rise to novel therapeutic targets.

\section{Acknowledgements:}

This work was supported in part by Carlos III Institute of Health, Spain (grant number PI18/00326), Fondo Europeo de Desarrollo Regional (FEDER), Unión Europea, "Una manera de hacer Europa". We are also indebted to the Neurological Tissue Bank of the Biobanc-Hospital Clínic-IDIBAPS (Barcelona) for providing human brain samples.

\section{References:}

1. You, X. et al. Neural circular RNAs are derived from synaptic genes and regulated by development and plasticity. Nat Neurosci. 18, 603-610 (2015).

2. Salta, E. \& de Strooper, B. Noncoding RNAs in Neurodegeneration. Nat Rev Neurosci. 18, 627640 (2017).

3. Rybak-Wolf, A. et al. Circular RNAs in the mammalian brain are highly abundant, conserved, and dynamically expressed. Mol Cell. 58, 870-885 (2015).

4. Dube, U. et al. An atlas of cortical circular RNA expression in Alzheimer disease brains demonstrates clinical and pathological associations. Nat Neurosci. doi. 10.1038/s41593-0190501-5. 
5. Gelpi E, et al. Phenotypic variability within the inclusion body spectrum of basophilic inclusion body disease and neuronal intermediate filament inclusion disease in frontotemporal lobar degenerations with FUS-positive inclusions. J Neuropathol Exp Neurol. 71, 795-805 (2012).

6. Montine TJ, et al. National Institute on Aging-Alzheimer's Association guidelines for the neuropathologic assessment of Alzheimer's disease: a practical approach. Acta Neuropathol 123, 1-11 (2012).

7. Cairns NJ, et al. Neuropathologic diagnostic and nosologic criteria for frontotemporal lobar degeneration: consensus of the Consortium for Frontotemporal Lobar Degeneration. Acta Neuropathol. 114, 5-22 (2007).

\section{Figure legend.}

cricRNA expression levels in each individual grouped by the five major phenotypes. Brain samples with pathogenic mutations in the FTLD-Tau and FTLD-TDP43 groups are depicted by a grey circle. * $P<0.05 ; * * P<0.01 ; * * *<0.001 ; * * * P<0.0001$. 
Table 1. Demographic, clinical and genetic data of all brain specimens.

\begin{tabular}{|c|c|c|c|c|c|}
\hline Case & Diagnosis & Gender & Age at death (y) & PMI, (h:m) & Mutations \\
\hline 1 & Control & Female & 68 & $13: 00$ & \\
\hline 2 & Control & Female & 81 & $23: 30$ & \\
\hline 3 & Control & Female & 83 & 07.30 & \\
\hline 4 & Control & Female & 83 & 07.30 & \\
\hline 5 & Control & Male & 64 & $10: 00$ & \\
\hline 6 & Control & Male & 78 & 06:00 & \\
\hline 7 & Control & Male & 31 & $17: 30$ & \\
\hline 8 & Control & Female & 50 & $12: 00$ & \\
\hline 9 & Control & Female & 37 & $13: 30$ & \\
\hline 10 & Control & Female & 74 & 03:40 & \\
\hline 11 & Control & Female & 56 & $14: 00$ & \\
\hline 12 & Control & Male & 71 & $<24: 00$ & \\
\hline 13 & Control & Female & 75 & $20: 00$ & \\
\hline 14 & Control & Female & 78 & $<24: 00$ & \\
\hline 15 & Control & Female & 77 & $20: 00$ & \\
\hline 16 & SAD & Male & 87 & 08:00 & \\
\hline 17 & SAD & Female & 83 & $20: 00$ & \\
\hline 18 & SAD & Male & 74 & $10: 00$ & \\
\hline 19 & SAD & Female & 74 & 14.33 & \\
\hline 20 & SAD & Female & 87 & 05:45 & \\
\hline 21 & SAD & Female & 75 & 04:00 & \\
\hline 22 & sAD & Female & 77 & 05:30 & \\
\hline 23 & SAD & Female & 83 & $10: 00$ & \\
\hline 24 & SAD & Female & 77 & $04: 30$ & \\
\hline 25 & SAD & Female & 86 & 09:00 & \\
\hline 26 & SAD & Male & 83 & 05:00 & \\
\hline 27 & SAD & Male & 75 & 04:15 & \\
\hline 28 & SAD & Female & 80 & 05.30 & \\
\hline 29 & SAD & Female & 73 & $14: 00$ & \\
\hline 30 & SAD & Female & 83 & $04: 30$ & \\
\hline 31 & SAD & Female & 61 & 04:30 & \\
\hline 32 & SAD & Female & 66 & 07:00 & \\
\hline 33 & SAD & Male & 56 & $16: 45$ & \\
\hline 34 & SAD & Male & 68 & 09:00 & \\
\hline 35 & fAD & Male & 57 & $15: 15$ & PSEN1 (p.M139T) \\
\hline 36 & fAD & Male & 44 & 05:30 & PSEN1 (p.E120G) \\
\hline 37 & fAD & Male & 57 & 09:30 & PSEN1 (p.V89L) \\
\hline 38 & fAD & Female & 56 & 05:00 & PSEN1 (p.L286P) \\
\hline 39 & fAD & Female & 56 & 06:00 & PSEN1 (p.P264L) \\
\hline 40 & fAD & Male & 64 & 14.45 & PSEN1 (p.M139T) \\
\hline 41 & fAD & Male & 36 & $15: 00$ & $A P P(p .1716 \mathrm{~F})$ \\
\hline 42 & fAD & Male & 53 & 05:15 & PSEN1 (p.M139T) \\
\hline 43 & fAD & Male & 68 & 06:10 & APP (duplication) \\
\hline
\end{tabular}




\begin{tabular}{|c|c|c|c|c|c|}
\hline 44 & FTLD-TDP43 & Female & 70 & $11: 00$ & \\
\hline 45 & FTLD-TDP43 & Male & 72 & $17: 30$ & \\
\hline 46 & FTLD-TDP43 & \begin{tabular}{|l|} 
Female \\
\end{tabular} & 69 & $11: 45$ & \\
\hline 47 & FTLD-TDP43 & Male & 65 & $13: 00$ & \\
\hline 48 & FTLD-TDP43 & Male & 77 & 06.20 & \\
\hline 49 & FTLD-TDP43 & Male & 73 & 13:00 & \\
\hline 50 & FTLD-TDP43 & Male & 71 & 07:00 & \\
\hline 51 & FTLD-TDP43 & Male & 61 & $07: 45$ & C9orf72 \\
\hline 52 & FTLD-TDP43 & Male & 66 & $15: 15$ & C9orf72 \\
\hline 53 & FTLD-TDP43 & Male & 69 & 05:00 & C9orf72 \\
\hline 54 & FTLD-TDP43 & Female & 69 & $13: 25$ & C9orf72 \\
\hline 55 & FTLD-TDP43 & Female & 58 & $11: 00$ & C9orf72 \\
\hline 56 & FTLD-TDP43 & Female & 57 & $04: 15$ & C9orf72 \\
\hline 57 & FTLD-TDP43 & Male & 55 & $12: 00$ & C9orf72 \\
\hline 58 & FTLD-TDP43 & Male & 69 & $05: 45$ & C9orf72 \\
\hline 59 & FTLD-TDP43 & \begin{tabular}{|l|} 
Female \\
\end{tabular} & 66 & $11: 30$ & C9orf72 \\
\hline 60 & PiD & Male & 74 & 07:00 & \\
\hline 61 & PiD & Male & 56 & 09:30 & \\
\hline 62 & PiD & Male & 74 & $10: 15$ & \\
\hline 63 & PiD & Female & 66 & $13: 30$ & \\
\hline 64 & CBD & Male & 58 & $13: 00$ & MAPT (p.P301L) \\
\hline 65 & CBD & Male & 58 & $10: 00$ & MAPT (p.P301L) \\
\hline 66 & CBD & Male & 53 & $16: 55$ & MAPT (p.P301L) \\
\hline 67 & CBD & Male & 72 & 05:50 & MAPT (p.P301L) \\
\hline 68 & CBD & Male & 75 & 05:55 & MAPT (p.P301L) \\
\hline 69 & CBD & Female & 63 & 07:15 & MAPT (p.P301L) \\
\hline
\end{tabular}

SAD: Sporadic Alzheimer's dementia; fAD: Familial Alzheimer's dementia; PiD: Pick's disease; CBD: Corticobasal degeneration; C9orf72 indicates patients with an expansion mutation of the GGGGCC hexanucleotide repeat (>30 repeats); PMI: Postmortem interval. 
Figure 1.
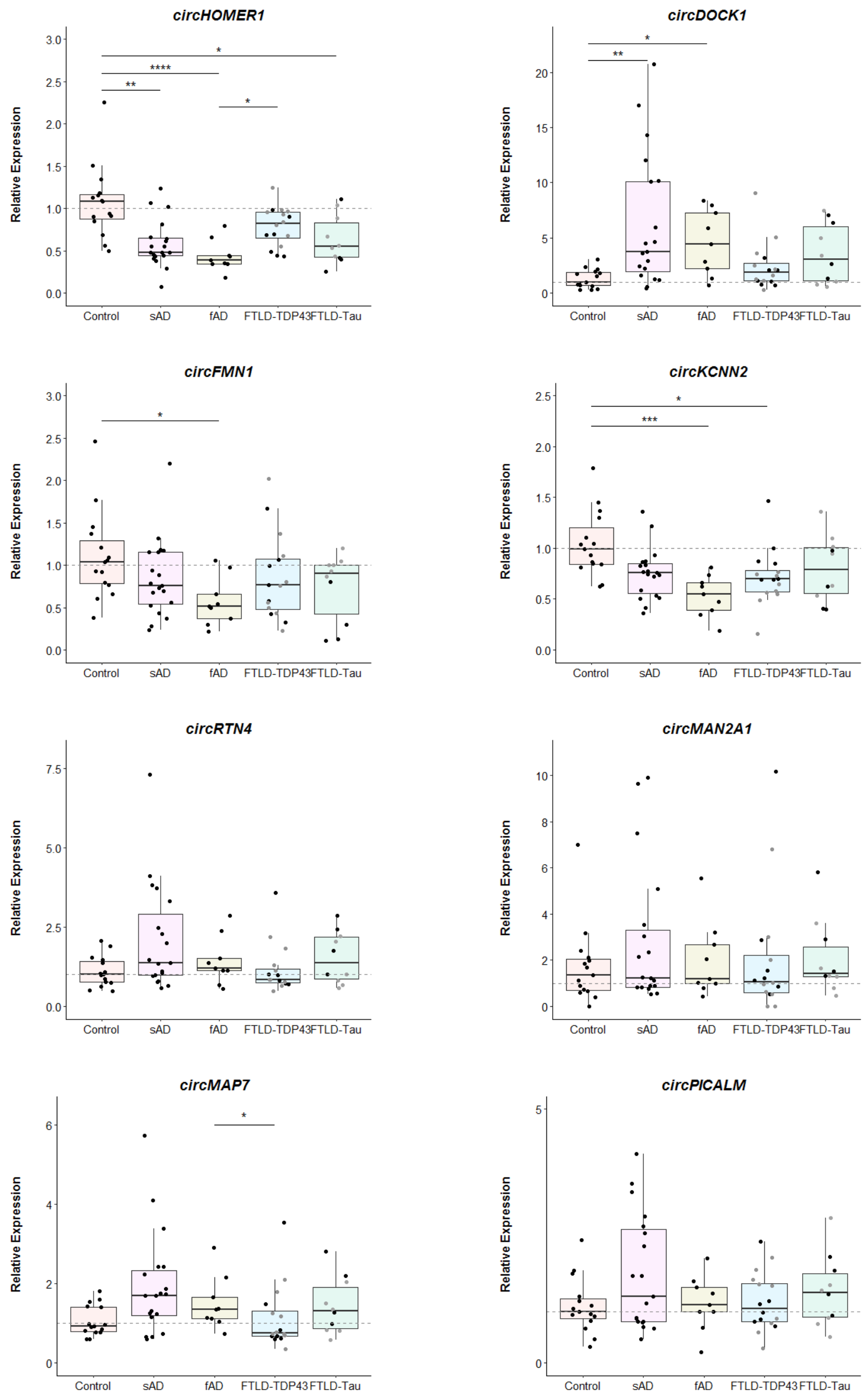